for the E2 transition between the $\frac{3}{2}^{-}$and $\frac{5}{2}^{-}$levels.

Besides these considerations some other arguments against the application of the core particle coupling model to $\mathrm{V}^{51}$ have been quoted (ref. 3) whereas the energies of the $\frac{5}{2}^{-}, \frac{3}{2}^{-}, \frac{1}{2}^{-}, \frac{9}{2}$ and $\frac{15^{-}}{2}$ levels in $\mathrm{V}^{51}$ are in agreement with the predictions of the three particles configuration model 18,19). However some problems remain unsolved when applyjing the three particles configuration model to $\mathrm{V}^{51}$, for instance the disagreement noted previously for the ratios and absolute values of the various $B$ (E2) with respect to the predictions of the three particles configuration model.

We thank Prof. M. Demeur and Dr. M. Nève de Mévergnies for helpfull discussions.

\section{References}

1) K. Way et al., Nuclear Data Sheets (National Academy of Sciences, National Research Council, Washington, U.S.A.).

2) J.E.Schwager, Phys. Rev. 121 (1961) 569.

3) H.W. Kendall and I. Talmi, Phys. Rev. 128 (1962) 792.
4) E.G.: J. P. Elliott and A. M. Lane, The nuclear shellmodel, in: Encyclopedia of physics, S. Flugge ed. (Springer-Verlag, Berlin, 1957) 39, p. 241.

5) H.E.Gove and C. Broude, Proc. Second Conf. on Reactions between complex nuclei, A. Zucker et al, eds (John Wiley and Sons, New York, 1960) p. 57.

6) Adams, Eccleshall and Yates, in:Reactions between complex nuclei (1960) p. 95.

7) I.K. Lemberg, in:Reactions between complex nuclei (1960) p. 112.

8) R. C. Ritter et al. , Phys. Rev. 128 (1962) 2320.

9) A. Braunstein and A.De-Shalit, Physics Letters I (1962) 264.

10) H.E. Gove, Physics Letters 4 (1963) 249.

11) J.Vervier, Nuovo Cimento, to be published.

12) A.De-Shalit, Phys.Rev. 122 (1961) 1530.

13) I. Y. Krause, Phys. Rev. 129 (1963) 1330.

14) S.A. Moszkowski, in:Beta- and gamma-ray spectroscopy, K. Siegbahn ed. (North Holland Publ. Co, Amsterdam, 1955) p. 373.

15) J.Vervier, to be published.

16) Grodzins, Bauer and Wilson, Phys. Rev. 124 (1961) 1897.

17) A.R. Edmonds and B.H. Flowers, Proc. Roy Soc. (London) A 214 (1952) 515.

18) R.D. Lawson and J. L. Uretsky, Phys.Rev. 106 (1957) 1369.

19) A.Dar (unpublished), quoted by A. De-Shalit, in : Selected topics in nuclear theory (I. A. E.A., 1963) p.209.

\title{
APPLICATION TO THE EXACT THEORY OF THE PAIRING MODEL TO SOME EVEN ISOTOPES OF LEAD *
}

\author{
R. W. RICHARDSON \\ H.M.Randall Laboratory of Physics, \\ University of Michigan, Ann Orbor, Michigan
}

Received 19 April 1963

In this note, we report the results of using the exact eigenstates of the pairing-force Hamiltonian in the pairing models of $\mathrm{Pb}^{206}, \mathrm{~Pb}^{204}$ and $\mathrm{Pb}^{202}$. The eigenstates of the models of these isotopes belong to the restricted class of eigenstates described in ref. 1). Using these eigenstates, we find that the pairing-interaction strength should be about $30 \%$ stronger than the value used in previous approximate calculations 2). This stronger pairing interaction plus the exact theory of the pairing model predict the observed pairing energies, within the experimental errors, and the excitation spectra (excluding the lowest $2^{+}$states), with an average error of $0.09 \mathrm{MeV}$ for these isotopes. The expressions for the wave function and energy of an eigenstate of the pairing-force Hamiltonian which we will use have been given in ref. 1). There, the unnormalized wave function and the energy of a state containing $2 N$ paired particles were given by

$$
\varphi\left(f_{1} \ldots f_{N}\right)=\sum_{P} P\left(\prod_{R=1}^{N}\left(2 \epsilon_{f_{R}}-E_{p_{R}}\right)^{-1}\right)_{(1)}
$$

and

$$
E=\sum_{i=1}^{N} E_{p_{i}},
$$

where $\epsilon_{f}$ is the energy of the single-particle state with quantum numbers $f, \Sigma_{P} P$ is a sum over the $N$. permutations $P$ of the indices $p_{1} \ldots p_{N}$, and * Supported by the National Science Foundation. 
the $N$ pair energies $E_{p_{i}}$ are roots of the $N$ coupled equations

$$
1=g_{i} \sum_{f}\left(2 \epsilon_{f}-E_{p_{i}}\right)^{-1}, i=1 \ldots N,
$$

where

$$
g_{i}=g\left(1+2 g \sum_{j \neq i} \frac{1}{E_{p_{j}}-E_{p_{i}}}\right)^{-1}
$$

The restriction on this class of states is that the pair energies must satisfy

$$
E_{p_{i}} \neq E_{p_{j}}, \text { all } i \neq j,
$$

in addition to (3).

In considering a specific system, the single particle spectrum $\epsilon_{f}$, and the pairing interaction strength, $g$, must be determined. For the isotopes of lead that we consider, we use the single-particle (hole) spectrum of $\mathrm{Pb}^{20}$ to determine the $\epsilon_{f}^{\prime} \mathrm{s}$ as was done in ref. 2). This spectrum is given in table 1. In this table, $\Omega_{n}=j+\frac{1}{2}$ is the pair degeneracy of the level $n$. In what follows, we will identify the levels by the index $n$ rather than $(l j)^{\pi}$. Thus, equation (3) now reads

$$
1=g_{i} \sum_{n=1}^{5} R_{n}\left(2 \epsilon_{n}-E_{p_{i}}\right)^{-1}
$$

for states containing no unpaired particles.

Table 1

The single-particle spectrum in $\mathrm{MeV}$

\begin{tabular}{|c|c|c|c|}
\hline$(l j)^{\pi}$ & $n$ & $\Omega_{n}$ & $e_{n}$ \\
\hline$\left(p \frac{1}{2}\right)^{-}$ & 1 & 1 & 0.00 \\
$\left(f \frac{5}{2}\right)^{-}$ & 2 & 3 & 0.57 \\
$\left(p \frac{3}{2}\right)^{-}$ & 3 & 2 & 0.90 \\
$\left(i \frac{13}{2}\right)^{+}$ & 4 & 7 & 1.63 \\
$\left(f \frac{7}{2}\right)^{-}$ & 5 & 4 & 2.35 \\
\hline
\end{tabular}

In order to determine $g$, we use the relation 1)

$$
\begin{aligned}
P_{n}(z, 2 N) & =2 E(z, 2 N-1)-E(z, 2 N)-E(z, 2 N-2) \\
& =2 \epsilon_{F}-E_{F}
\end{aligned}
$$

between the observed pairing energy, $P_{n}$, and the pair energy $E_{F}$. This, together with the mass data given by Everling et al. 3), can be used to determine $E_{F}$. Eqs. (6) then determine the remaining $N-1$ ground-state pair energies and the interaction strength $g$. However, $P_{n}$ is not known accurately enough to determine $g$ uniquely and interaction strengths in the range $0.135<g<0.165 \mathrm{MeV}$ will reproduce the observed $P_{n}^{\prime}$ s. We ther efore pick the value of $g$ in this range which reproduces the excitation spectra best. This leads to the value $g=$ $0.146 \mathrm{MeV}$. Note that this is considerably stronger than the value, $g=0.111 \mathrm{MeV}$, which has been used in previous calculations 2).

With $\epsilon_{f}$ and $g$ determined, all the parameters of the model are fixed and there remains the solution of (3) for the $E_{p_{i}}$. The solutions and the excitation energies for some states of $\mathrm{Pb}^{206}, \mathrm{~Pb}^{204}$ and $\mathrm{Pb}^{202}$ are given in tables 2, 3 and 4. We identify the states by giving their spin and parity and the configurations to which they correspond in the limit $g \rightarrow 0$ (config.). We also list the observed levels of these nuclei 4) (Exp.) next to the states of the model with the same spins and parities which best reproduce the observed excitation energies. The pair energies given are the roots of (3) which we now discuss separately for the three systems.

Table 2

Excitation spectrum of $\mathrm{Pb}^{206}$ in $\mathrm{MeV}$

\begin{tabular}{|c|c|c|c|c|}
\hline (spin) & Config. & Exp. & Model & Pair energies \\
\hline$(0)^{+}$ & $(1)^{2}$ & 0.00 & 0.00 & $E_{1}=-0.59$ \\
$(0)^{+}$ & $(2)^{2}$ & $(1.19)$ & 1.03 & $E_{2}=0.44$ \\
$(1)^{+}$ & $(1)(3)$ & $(1.72)$ & 1.49 & - \\
$(2)^{+}$ & $(1)(2)$ & 0.80 & 1.16 & - \\
$(2)^{+}$ & $(1)(3)$ & 1.47 & 1.49 & - \\
$(2)^{+}$ & $(2)^{2}$ & 1.85 & 1.73 & - \\
$(3)^{+}$ & $(1)(2)$ & 1.34 & 1.16 & - \\
$(4)^{+}$ & $(2)^{2}$ & 1.68 & 1.73 & - \\
$(4)^{+}$ & $(2)(3)$ & 2.00 & 2.06 & - \\
$(4)^{+}$ & $(1)(5)$ & $(2.95)$ & 2.94 & - \\
$(5)^{-}$ & $(2)(4)$ & $(2.78)$ & 2.79 & - \\
$(5)^{-}$ & $(3)(4)$ & 3.02 & 3.12 & - \\
$(6)^{-}$ & $(1)(4)$ & $(2.38)$ & 2.22 & - \\
$(7)^{-}$ & $(1)(4)$ & $(2.20)$ & 2.22 & - \\
\hline
\end{tabular}

Table 3

Excitation spectrum of $\mathrm{Pb}^{204}$ in $\mathrm{MeV}$

\begin{tabular}{|c|c|c|c|l|}
\hline (spin) & Config. & Exp. & Model & \multicolumn{1}{|c|}{ Pair energies } \\
\hline$(0)^{+}$ & $(1)^{2}(2)^{2}$ & 0.00 & 0.00 & $\xi=-0.30, \eta^{2}=0.15$ \\
$(0)^{+}$ & $(2)^{4}$ & - & 1.48 & $5=0.44, \eta^{2}=0.093$ \\
$(2)^{+}$ & $(1)^{2}(2)^{2}$ & 0.90 & 1.35 & $E_{1}=-0.39$ \\
$(4)^{+}$ & $(1)^{2}(2)$ & 1.27 & 1.35 & $E_{1}=-0.39$ \\
$(4)^{+}$ & $(1)^{2}(2)(3)$ & 1.56 & 1.66 & $E_{1}=-0.41$ \\
$(9)^{-}$ & $(1)^{2}(2)(4)$ & 2.19 & 2.30 & $E_{1}=-0.44$ \\
\hline
\end{tabular}

Table 4

Excitation spectrum of $\mathrm{Pb}^{202}$ in $\mathrm{MeV}$

\begin{tabular}{|c|l|c|c|c|}
\hline (spin) & Config. & Exp. & Model & \multicolumn{1}{|c|}{ Pair energies } \\
\hline$(0)^{+}$ & $(1)^{2}(2)^{4}$ & 0.00 & 0.00 & $E_{1}=-0.30, \xi=0.06, \eta^{2}=0.498$ \\
$(0)^{+}$ & $(1)^{2}(2)^{2}(3)^{2}$ & - & 1.76 & $E_{1}=-0.12, E_{2}=0.17, E_{3}=1.53$ \\
$(2)^{+}$ & $(1)^{2}(2)^{4}$ & 0.96 & 1.42 & $E_{1}=-0.13, E_{2}=0.23$ \\
$(4)^{+}$ & $(1)^{2}(2)^{4}$ & 1.38 & 1.42 & $E_{1}=-0.13, E_{2}=0.23$ \\
$(4)^{+}$ & $(1)^{2}(2)^{3}(3)$ & 1.62 & 1.62 & $\xi^{=-0.015,} \eta_{2=0.00925}^{2}$ \\
$(5)^{-}$ & $(1)^{2}(2)^{3}(4)$ & 2.04 & 2.26 & $\xi=-0.06, \eta^{2}=0.035$ \\
$(9)^{-}$ & $(1)^{2}(2)^{3}(4)$ & 2.19 & 2.26 & $\xi=-0.06, \eta^{2}=0.035$ \\
\hline
\end{tabular}


The solution of eq. (3) for $\mathrm{Pb}^{206}$ is quite simple since, in this case, they reduce to the single equation

$$
1=g \sum_{n} \Omega_{n}\left(2 \epsilon_{n}-E_{p}\right)^{-1}
$$

for the one unknown, $E_{p}$, and the restrictions (5), play no rule. Two roots, $E_{1}$ and $E_{2}$, of eq. (8) are given in table 2 and they correspond to the energies of the ground and first excited $0^{+}$states of $\mathrm{Pb}^{206}$. The energies of the remaining states, which do not contain any paired particles, are given by $\epsilon_{l}+\epsilon_{m}$, where $l$ and $m$ are the single-particle states which are occupied.

For $\mathrm{Pb}^{204}$, study of eq. (3) indicates that for $g>0.115 \mathrm{MeV}$ the two pair energies of the ground state are complex and in fact are complex conjugates of each other. This is also true for the state $(2)_{0}^{4}$ for $g<0.209 \mathrm{MeV}$. We ther efore represent the two pair energies of these states as

$$
E_{1,2}=\xi \pm \text { in }
$$

Equating the real and imaginary parts of eq. (3) then gives the equations

$$
\begin{aligned}
& 1=g \sum_{n} \frac{\Omega_{n}\left(2 \xi_{n}-\xi\right)}{\left(2 \epsilon_{n}-\xi\right)^{2}+n^{2}} \\
& 1=\eta^{2} \sum_{n} \frac{\Omega_{n}}{\left(2 \epsilon_{n}-\xi\right)^{2}+\eta^{2}}
\end{aligned}
$$

for $\xi$ and $\eta$. The restrictions (5), for these states are $\eta \neq 0$. The energies are then given by (2)

$$
E=E_{1}+E_{2}=2 \xi \text {. }
$$

In table 3, we give the values of $\xi$ and $\eta^{2}$ for these two states for $g=0.146 \mathrm{MeV}$.

The remaining states of $\mathrm{Pb}^{204}$ which we treat contain two paired and two unpaired particles. The eq. (3) for these states are the same as (8) with the one change that $\Omega_{n}$ is replaced by $\Omega_{n}-\delta_{n l}-\delta_{n m}$, where $l$ and $m$ are the states occupied by the unpaired particles. The energies of these states are given by

$$
E=\epsilon_{l}+\epsilon_{m}+E_{p} .
$$

For the ground state of $\mathrm{Pb}^{202}$, study of eq. (3) indicates that one pair energy $E_{1}$, is real and two pair energies $E_{2}$ and $E_{3}$, are complex as in (9). Equating the real and imaginary parts of eq. (3) then yields the equations

$$
1+\frac{4 g\left(\xi-E_{1}\right)}{\left(\xi-E_{1}\right)^{2}+\eta^{2}}=g \sum_{n} \Omega_{n}\left(2 \epsilon_{n}-E_{1}\right)^{-1}
$$

$$
\begin{aligned}
& 1-\frac{2 g\left(\xi-E_{1}\right)}{\left(\xi-E_{1}\right)^{2}+\eta^{2}}=g \sum_{n} \frac{\Omega_{n}\left(2 \epsilon_{n}-\xi\right)}{\left(2 \epsilon_{n}-\xi\right)^{2}+\eta^{2}} \\
& 1+\frac{2 \eta^{2}}{\left(\xi-E_{1}\right)^{2}+\eta^{2}}=\eta^{2} \sum_{n} \frac{\Omega_{n}}{\left(2 \epsilon_{n}-\xi\right)^{2}+\eta^{2}}
\end{aligned}
$$

for $E_{1}, \xi$ and $\eta$. Again, the restrictions (5) become $\eta \neq 0$. The energy of this state is given by (2)

$$
E=E_{1}+E_{2}+E_{3}=E_{1}+2 \xi .
$$

For the other $0^{+}$state that we treat, the pair energies are real and may be obtained from a straightforward solution of eq. (3).

The remaining states of $\mathrm{Pb}^{202}$ that we treat contain four paired and two unpaired particles. For the states $(1)^{2}(2)_{2}^{4}, 4^{+}$, the two pair energies are real and may be obtained by solving eq. (3) using $\Omega_{n}-2 \delta_{n 2}$ instead of $\Omega_{n}$ The energies of these states are then given by

$$
E=2 \epsilon_{2}+E_{1}+E_{2} \text {. }
$$

For the other excited states, the pair energies have the form (9), where $\xi$ and $\eta$ satisfy (10) and (11) with $\Omega_{n}$ replaced by $\Omega_{n}-\delta_{n l}-\delta_{n m}$. The energies of these states are given by

$$
E=\epsilon_{l}+\epsilon_{m}+2 \xi \text {. }
$$

If we exclude the lowest $2^{+}$states of these nuclei from our consideration (since these are collective levels 2 ), we obtain an average error of $0.09 \mathrm{MeV}$ in the predicted excitation spectra. This is about $\frac{1}{4}$ the average error in the approximate treatment of the model given in ref. 2 ).

In addition to the excitation energies of the states, we have given the pair energies in tables 2,3 and 4 . These may be inserted in (1) to obtain the model wave function. The matrix elements of any operator may then be calculated using these exact model wave function.

The details of these calculations will be reported at a later date.

\section{References}

1) R.W. Richardson, Physics Letters 3 (1963) 277.

2) L.S. Kisslinger and R. A. Sorensen, Dan. Mat. Fys Medd. 32, No. 9 (1960).

3) F. Everling, et al., Nuclear Phys. 18 (1960) 529.

4) K. Way et al., Energy levels of nuclei : $A=5$ to $A=257$ (Landolt-Bornstein Numerical Data and Functional Relationships in Science and Technology, New Series, Group I; Berlin: Springer Verlag, 1961), Vol.1 